\title{
Application of Fractal Grids in Industrial Low-Swirl Combustion
}

\author{
G. D. ten Thij ${ }^{1}$ - A. A. Verbeek ${ }^{1}$ T. H. van der Meer ${ }^{1}$
}

Received: 8 May 2015 / Accepted: 5 October 2015 / Published online: 27 October 2015

(C) The Author(s) 2015. This article is published with open access at Springerlink.com

\begin{abstract}
Fractal-grid-generated turbulence is a successful technique to significantly increase the reaction rate in the center of a low-swirl flame. Previous results (Verbeek et al. Combust. Flame 162(1), 129-143, 2015) are promising, but the experiments are only performed using natural gas at a single equivalence ratio and flow rate. In industry, the need arises to adapt gas turbines to a wider range of fuels, such as biogas and syngas. To simulate these other fuels, natural gas is enriched with up to $30 \%$ hydrogen (molar based). By means of planar OH-LIF, the turbulent flame speed is assessed. It is shown that the beneficial effect of fractal-grid-generated turbulence remains upon hydrogen enrichment. The fractal grids enhance the combustion in an energy efficient way, irrespective of the hydrogen fraction. Moreover, the characteristic linear relation of the normalized local consumption speed versus the normalized rms velocity holds for the investigated range, with an increasing coefficient upon hydrogen enrichment. For industry, a wide operability range is essential to operate at part load, therefore the lean stability limit is investigated, as well. It is shown that fractal grids increase the lean stability limit, i.e., the adiabatic flame temperature at which blow off occurs, by $50 \mathrm{~K}$, compared to a standard grid. Increasing the bulk flow significantly increases the lean stability limit and the difference between the two investigated grid types increases upon hydrogen enrichment. Hydrogen addition results in a decrease in the lean stability limit, regardless of the grid. A positive correlation was found between the adiabatic flame temperature at blow-off and the rms velocity at the flame brush. The outcome of the presented study provides, despite a slightly increased lean stability limit, a promising prospect for the application of fractal grids in industrial low-swirl combustion.
\end{abstract}

\footnotetext{
A. A. Verbeek

a.a.verbeek@utwente.nl

G. D. ten Thij

guidotenthij@gmail.com

1 Laboratory of Thermal Engineering, Faculty CTW, Twente University, PO Box 217, 7500AE,

Enschede, The Netherlands
} 
Keywords Fractal-grid-generated turbulence · Low-swirl burner · Hydrogen-enriched natural gas · Local consumption speed · Lean stability limit · Lean premixed combustion

\section{Introduction}

Low-swirl combustion has proven to be a promising technique that can result in a significant reduction of NOx emissions for lean premixed combustion [1-3]. A premixed flame is stabilized in mid air unattached from the burner geometry. Especially the center of a low-swirl flame can be considered as a freely propagating flame [4] for which the turbulent flame speed is dominated by the turbulence present in the upstream flow [5-7].

In the majority of the previous work the turbulence is varied by changing the mean flow rate, thereby keeping a constant turbulent intensity. Verbeek et al. [8] used fractal-gridgenerated turbulence to increase the level of turbulence, while maintaining a constant flow rate. Although the use of fractal grids to enhance the rate of combustion is not new, earlier work tends to focus on academic flames like a counter flow flames [9] or a V-shaped flames [10] whereas Verbeek et al. [8] use an industrial burner. It is shown that fractal grids are a successful technique to significantly increase the limited reaction rate in the center of the low-swirl flame. However, this study is only performed using natural gas at a single flow rate and equivalence ratio.

To apply low-swirl burners equipped with fractal grids in industry, more research is needed towards more industrial operating conditions. As a first step multiple levels of hydrogen enrichment were studied to simulate alternative fuels like syngases and biogases. And moreover, a study is performed to evaluate the effect on the lean stability limit, i.e., a measure for the achievable turn-down ratio of a premixed gas turbine engine.

The results are two-fold. Using fractal grids enhances the combustion in an efficient way, irrespective of the hydrogen fraction, at all studied conditions. However, using fractal grids reduces somewhat the achievable turn-down ratio (as it increases the lean stability limit).

A fractal grid consists of a geometric pattern that is constructed according to an iterative multi-scale pattern. Here, the 'cross' pattern is regarded because it is compatible with a lowswirl burner due to its near-constant velocity profile as a function of the radial direction. The turbulence created by these fractal grids is found to be more intense than turbulence created by classical grids [11]. This effect can be used in combustion [8-10] but also in many other applications, see e.g., [12-14]. The higher rms (root mean square) velocity upstream of fractal grids is explained by this extension of the turbulence production region [15]. Three of the fractal grids from Verbeek et al. [8] are used in this study and are shown in Fig. 1. These grids have a blockage of $60 \%$. The thickness ratio, $R_{\mathrm{t}}$, is defined as the ratio of
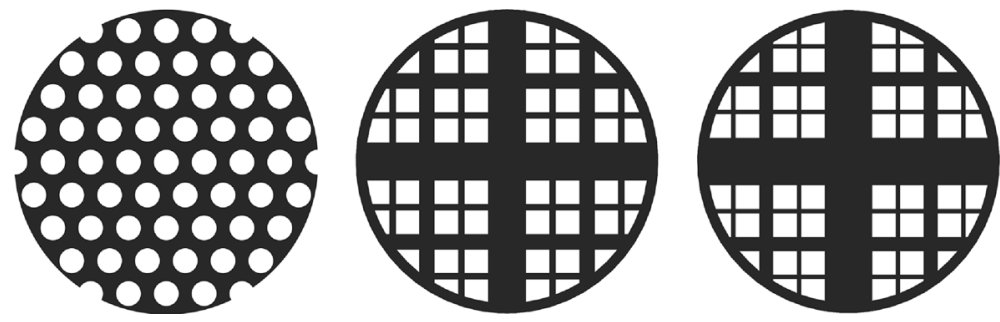

Fig. 1 Turbulence grids that are used in experiments. L-R: Hexagonal, Fractal $R_{\mathrm{t}}=0.40$ and $R_{\mathrm{t}}=0.29$ grids 
(a)

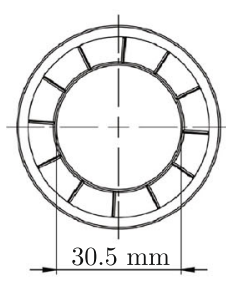

(b)

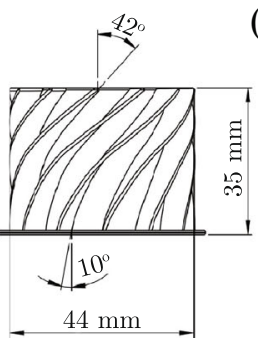

(c)

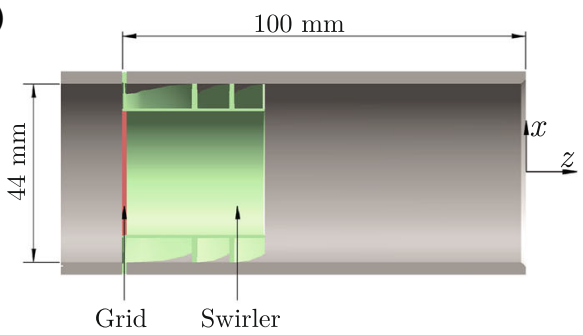

Fig. 2 Component overview of the burner: a Top view of the swirler, b Side view of the swirler and c Sectional plane. The flow in the sectional plane is from left to right. On the right the coordinate system is given, such that $z=0$ is at the exit of the burner. The flame front is located at approximately $z=20 \mathrm{~mm}$

two consecutive bar-thicknesses. The grids are positioned inside the swirler geometry, as indicated in Fig. 2.

In the industry, natural gas is most often used as fuel in power-generating gas turbines. Due to the finiteness of fossil fuels, the need arises to cost-effectively utilize gaseous alternatives, e.g., mixtures that are produced in coal gasification installations, biomass-derived fuels or chemical plant by-product gases. The molar based hydrogen content of these future fuels range from a few percentage to over $60 \%$ [16]. Blending these hydrogen-rich fuels with natural gas provides both a solution to the immediate need for NOx emission reduction, and a transition strategy to a carbon-free energy system in the future [17]. Mixing these syngases with natural gas is non-trivial, because it alters the combustion properties in terms of flame speed and flammability limits significantly [18]. These alterations originate particularly from the addition of hydrogen [19].

It has already been shown that low-swirl mode of combustion is suitable for hydrogen rich fuels, e.g, [20-22], which results in even lower NOx emissions compared to methane. The lean stability limit is reduced due to the higher reactivity of hydrogen and a higher air excess is possible, thereby lowering adiabatic flame temperature and the associated NOx emissions. The current work tests if these properties are maintained when the flame is exposed to a flow with a higher turbulence intensity generated by fractal grids.

The first objective is to determine how hydrogen enrichment of natural gas influences the beneficial combination of fractal grids and low-swirl combustion, which is referred to as the Bouten effect. This objective is researched at multiple hydrogen fractions and at a constant equivalence ratio $(\phi=0.7)$. The second objective is to determine how fractal-gridgenerated turbulence influences the lean stability limit. This is evaluated at several flow rates and multiple hydrogen fractions.

The organization of this paper is as follows. In Section 2 the three experimental methods are explained. Section 3 is devoted to the first objective and in Section 4 the second objective is discussed. Concluding remarks are made in Section 5.

\section{Experimental Methods}

\subsection{Measuring flame front}

The flames are studied using OH-LIF, which provides instantaneous 2D cross sectional images of the flame. A statistical canalysis of the flame front is obtained by calculating the 
mean progress variable, flame surface density (FSD) and local consumption speed. First, a subsection describes the equipment that is used. In Section 2.1.2, the method that is used to detect the flame front is shortly described. In Section 2.1.3, two methods for calculating the local consumption speed are discussed.

\subsubsection{Equipment}

In this study, the same equipment is used as by Verbeek et al. [8]. An excimer pumped dye laser (Lambda Physik LPX 240i in combination with Lambda Physik Scanmate 2, Coumarin-153 dye dissolved in Methanol) is used to generate light with a wavelength $283.010 \mathrm{~nm}$. A series of 5 lenses is used to convert the laser beam into a sheet with a height of approximately $80 \mathrm{~mm}$ and a thickness less than $0.150 \mathrm{~mm}$. An ICCD camera (PI-MAX 3 ) with a resolution of $1024 \times 1024 \mathrm{px}$ is used to capture the OH-LIF signal.

\subsubsection{Flame front extraction}

The flame front extraction method is identical as used by Verbeek et al. [8, 23]. An edge detection algorithm is used to extract the flame front geometry from the OH-LIF images. The algorithm uses a combination of two edge-detectors, i.e., Canny and Laplacian of Gaussian (LoG), to determine the flame front from the OH-LIF data. The correct edges are found by setting a threshold value for the overlap of these two methods. A piecewise polynomial is fitted through the found edge for a smooth representation of the flame front.

\subsubsection{Local consumption speed}

The OH-LIF experiments have been carried out to quantify the local consumption speed, $S_{\mathrm{T}, \mathrm{LC}}$. In order to calculate $S_{\mathrm{T}, \mathrm{LC}}$, the mean progress variable, $\bar{c}$, and the flame surface density, $\Sigma_{3 \mathrm{D}}$, are required. Since these are only intermediary results, they are only briefly discussed here. A more complete clarification is given by Verbeek et al. [8]. The mean progress variable expresses the probability that the mixture at a certain point is burned. It is determined by averaging the instantaneous reaction progress, obtained from the instantaneous binarized images, where $c=0$ denotes a region that contains unburned mixture and $c=1$ denotes a region that is burned. The flame surface density is defined as the time-averaged surface-area in an infinitesimal box. The local consumption speed has been calculated using Eq. 1, where $\eta$ is the coordinate perpendicular to the flame brush and $I_{0}$ is the stretch factor which relates the averaged local consumption speed of the flamelet and the laminar unstretched flame speed; $I_{0}=\bar{S}_{\mathrm{F}, \mathrm{C}} / S_{\mathrm{L} 0} . S_{\mathrm{L} 0}$ has been calculated using CHEMKIN PREMIX and the GRI 3.0 reaction mechanism [24].

$$
\begin{aligned}
\frac{S_{\mathrm{T}, \mathrm{LC}}}{S_{\mathrm{L} 0}} & =I_{0} \int_{-\infty}^{\infty} \bar{\Sigma}_{3 \mathrm{D}} \mathrm{d} \eta \\
\Sigma_{3 \mathrm{D}} & =\frac{\Sigma_{2 \mathrm{D}}}{\langle\cos \psi\rangle}=\frac{\lim _{\Delta x \rightarrow 0} \frac{\bar{L}_{\mathrm{f}}}{(\Delta x)^{2}}}{\langle\cos \psi\rangle}
\end{aligned}
$$

The three dimensional flame surface density is given in Eq. 2, where $\Sigma_{2 \mathrm{D}}$ is defined as the time-averaged flame front length, $\bar{L}_{\mathrm{f}}$, within an infinitesimal area, $(\Delta x)^{2}$. The method of Veynante et al. [25] has been used to estimate the 3D FSD from the 2D measurements, by means of a conversion factor, $\langle\cos \psi\rangle$, which is based on the axial similarity of the in-plane fluctuations and the fluctuations out of the measurement plane. The conversion variable, $\psi$, 
is the angle between the unit vector normal to the instantaneous three-dimensional flame surface and the measurement plane. A stretch factor of unity is assumed in this study, which, based on Driscoll [26], is valid for methane flames. Although this is a somewhat cumbersome assumption for hydrogen-enriched natural gas, it is made only due to lack of a better alternative. The aim of this method is to asses the local consumption speed in the center of the flame, not on the centerline. Therefore, $\Sigma_{3 \mathrm{D}}(x, z)$ has been averaged in radial direction up to a radius of $x=5 \mathrm{~mm}$, which results in $\bar{\Sigma}_{3 \mathrm{D}}(z)$. Here, $z$ is in the flow direction and $x$ is in the radial direction in the measurement plane, see Fig. 2. This averaged flame surface density is converged when using at least 1000 frames. Equation 1 states that the flame surface density should then be integrated over the entire perpendicular coordinate $(z)$. If the entire flame is not captured for all cases, an appropriate range in terms of $\bar{c}$ has to be chosen in order to make a proper comparison between the different cases. Driscoll [26] states that the definition of flame speed is precise, unambiguous, but it involves an arbitrary choice of this range in $\bar{c}$. The range in $\bar{c}$ over which $S_{\mathrm{T}, \mathrm{LC}}$ has been calculated here is explained below.

Bouten effect for hydrogen enrichment Two methods are commonly used to calculate $S_{\mathrm{T}, \mathrm{LC}}$ from $\bar{\Sigma}_{3 \mathrm{D}}$; integration using Eq. 1 or parameterizing using Eq. 3, where $\Sigma_{\max }$ is the maximum FSD according to the quadratic fit: $\Sigma=4 \Sigma_{\max } \bar{c}(1-\bar{c})$. The turbulent flame brush thickness, $\delta_{\mathrm{T}}$, follows from parameterizing the mean reaction progress as $\bar{c}=$ $\left[1+\exp \left(\left(-4\left(\eta-\eta_{0.5}\right) / \delta_{\mathrm{T}}\right)\right]^{-1}\right.$, where $\eta_{0.5}$ is the value of $\eta$ at which $\bar{c}=0.5$.

$$
\frac{S_{\mathrm{T}, \mathrm{LC}}}{S_{\mathrm{L} 0}}=I_{0} \Sigma_{\max } \delta_{\mathrm{T}}
$$

Figure 3a visualizes the integration method, where $\bar{\Sigma}_{3 \mathrm{D}}$ is plotted versus the coordinate perpendicular to the flame brush together with two vertical lines representing the locations $\bar{c}=0.15$ and 0.85 . The cases that are not shown here contain similar results. The figure shows that integration of part of the flame brush (e.g., $0.15 \leq \bar{c} \leq 0.85$ ) under-predicts $S_{\mathrm{T}, \mathrm{LC}}$, because not the whole flame region is used in Eq. 1. Therefore the entire flame brush should be used for integration. In Fig. $3 b, \bar{\Sigma}_{3 \mathrm{D}}$ is plotted along with the parameterized curve according to Eq. 3 (dashed line), which shows that the fitted curve does not overlap well with the data. This is because it assumes a symmetric $\bar{\Sigma}_{3 D}$ profile, which is clearly not the case.
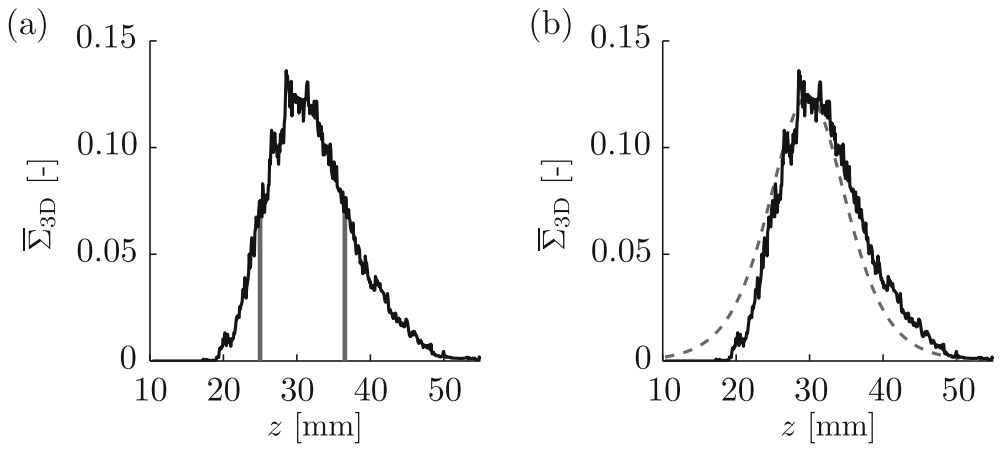

Fig. 3 Averaged flame surface density (in radial direction up to a radius of $x=5 \mathrm{~mm}$ ) versus coordinate $z$ for hexagonal grid, $n_{H_{2}}=0$ and $Q_{\text {tot }}=40 \mathrm{~m}_{\mathrm{n}}^{3} / \mathrm{h}$ for a integration method (1) where the gray lines equal $0.15 \leq \bar{c} \leq 0.85$ range limits and $\mathbf{b}$ parameterization method (3) where dashed line is the fitted FSD 


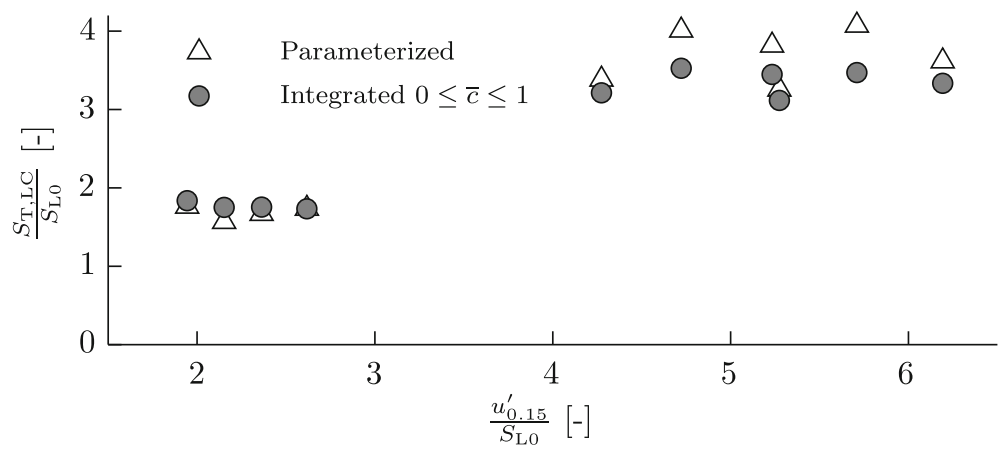

Fig. 4 Local consumption speed versus rms velocity at the flame brush for the parameterizing method and integrated over entire flame brush $(0 \leq \bar{c} \leq 1)$

In Fig. 4, the difference between the two methods can be seen. The four points at low $u_{0.15}^{\prime} / S_{\mathrm{L} 0}$ are the cases with the hexagonal grid. The parameterized data overlaps well with the data integrated over the entire flame brush for the hexagonal grid. For the fractal grids, parameterizing over-predicts the turbulent local consumption speed. This confirms that parameterizing is unsatisfactory for the investigated parameter range. Therefore, the turbulent local consumption speed has been determined by integrating over the total flame brush.

Effect on lean stability limit A different approach is required for the processing of the results at the lean stability limit. In contrary to standard $\bar{c}$ development (a monotonic increase from zero to unity), here the mean progress variable increases to a maximum, $\bar{c}_{\max }$, which is below unity, after which it decreases again.

Close to blow-off the combustion is limited to the center region in kernel-like structures. Figure $5 \mathrm{~b}$ illustrates this fact. The complete edge of the kernels are considered as reactive layers, much similar to the data presented by Kariuki et al. [27]. The sharp transition in intensity at the trailing edge supports the fact that it should be considered as a flame front. This is different from the drop in $\mathrm{OH}$ intensity somewhat downstream of the flame front as observed for pure $\mathrm{H}_{2}$ flames by Day et al.[28]. In fact, they used an additional step in their edge-detection algorithm to suppress edges detected downstream of the flame front based on a low intensity change.

Large portions of cold reactants escape unburned alongside. In the downstream region this causes $\bar{c}$ to reduce, due to the entrainment of this unburned mixture. This results in

(a)

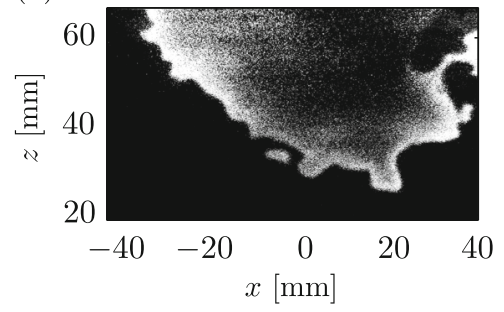

(b)

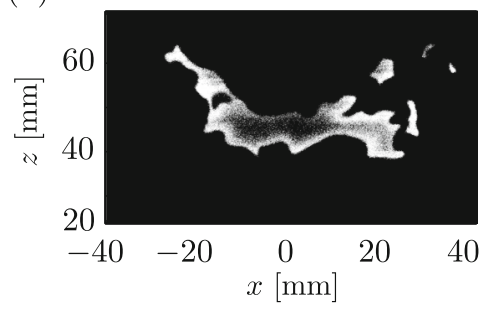

Fig. 5 Examples of the $\mathrm{OH}$-intensity images using the hexagonal grid at $\mathbf{a} \phi=0.7$ and $\mathbf{b}$ lean stability limit 
(a)

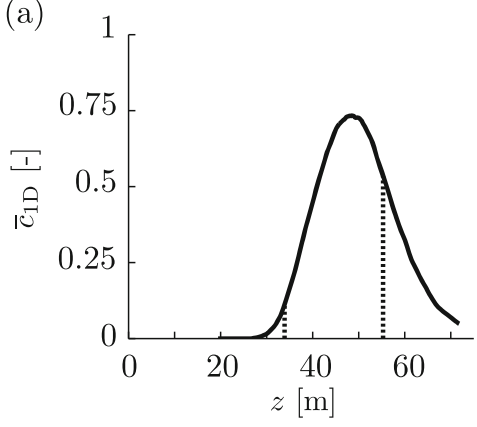

(b)

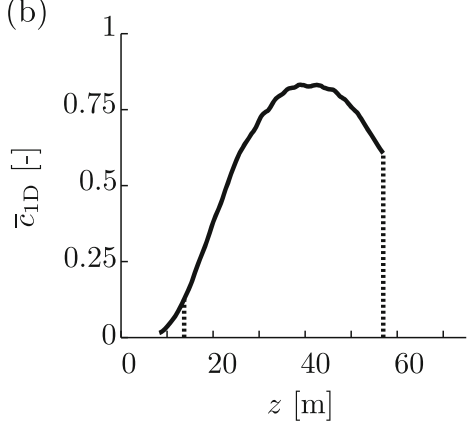

Fig. 6 Mean progress variable (averaged in radial direction up to a radius of $x=5 \mathrm{~mm}$ ) versus $z$ for a Hexagonal grid, $n_{H_{2}}=0$ and $Q_{\text {tot }}=40 \mathrm{~m}_{\mathrm{n}}^{3} / \mathrm{h}$ b Fractal grid $\left(R_{\mathrm{t}}=0.29\right), n_{H_{2}}=0$ and $Q_{\mathrm{tot}}=40 \mathrm{~m}_{\mathrm{n}}^{3} / \mathrm{h}$. The dotted lines show the boundaries of the interpolation range. The shown data is at the lean stability limit

varying ranges of $\bar{c}$ that are captured over the experiments; $\bar{c}$ at the upstream-cameraboundary is approximately zero for all cases, $\bar{c}_{\max }$ ranges from 0.4 to 0.8 and $\bar{c}$ at the downstream-camera-boundary ranges from zero to 0.6. In Fig. 10 an example can be found.

The plots are similar in shape, but have a different maximum. Therefore, the only way to define equivalent ranges of $\bar{c}$, is to make it dependent on $\bar{c}_{\max }$. The common range is $\bar{c}=0.15 \bar{c}_{\max }$ to $\bar{c}=0.73 \bar{c}_{\max }$ (respectively up- and downstream of the maximum), which is shown in Fig. 6a. The limiting case is shown in Fig. 6b. This equivalent range of $\bar{c}$ will be used to calculate the flame speed for the effect on the lean stability limit. By not integrating over the entire flame brush, i.e. $0 \leq \bar{c} \leq 1$, we underestimate $S_{\mathrm{T}, \mathrm{LC}}$, as discussed before. Although $S_{\mathrm{T}, \mathrm{LC}}$ is underestimated, this method provides a valuable, proper comparison, which is the aim of the method.

\subsection{Measuring turbulence at the flame brush}

Constant temperature hot-wire anemometry has been used to determine the velocity field at the flame. A locally manufactured, single probe of $5 \mu \mathrm{m}$ diameter platinum coated tungsten wire with a length of $0.9 \mathrm{~mm}$ has been used in combination with a Dantec $90 \mathrm{C} 10$ system. More details about the setup can be found in [29]. The velocity data is sampled at a frequency of $1 \mathrm{kHz}$ for $10 \mathrm{~s}$ to obtain converged results [8]. The velocity is split into a mean, $\bar{U}$, and a fluctuating part, $u$, by means of Reynolds decomposition. The root mean square (rms) of the fluctuating velocity, $u^{\prime}$, is also known as the rms velocity [30]. The hot-wire measurement method has a finite precision, which depends on the turbulence intensity, $u^{\prime} / \bar{U}$. Based on the findings of Swaminathan et al. [31], the error in $\bar{U}$ is above $5 \%$ for turbulent intensities higher than $30 \%$. In order to have a single measurement value for the rms velocity at the flame brush, the centerline value of the rms velocity will be used. The cold flow data will be used to quantify the flow field at combustion. Due to thermal expansion, the cold flow data does not match the data of the flow with combustion. However, for the leading edge of the flame, this effect is minimal. Therefore, the leading edge of the flame will be used for the calculation of the rms velocity at the flame brush. The leading edge of the flame is here defined at $\bar{c}=0.15$, therefore the rms velocity at the flame brush is denoted as $u_{0.15}^{\prime}$. Choosing a different value of $\bar{c}$ does not change the relation between the rms velocities significantly and it does not influence the conclusions at all. The difference 

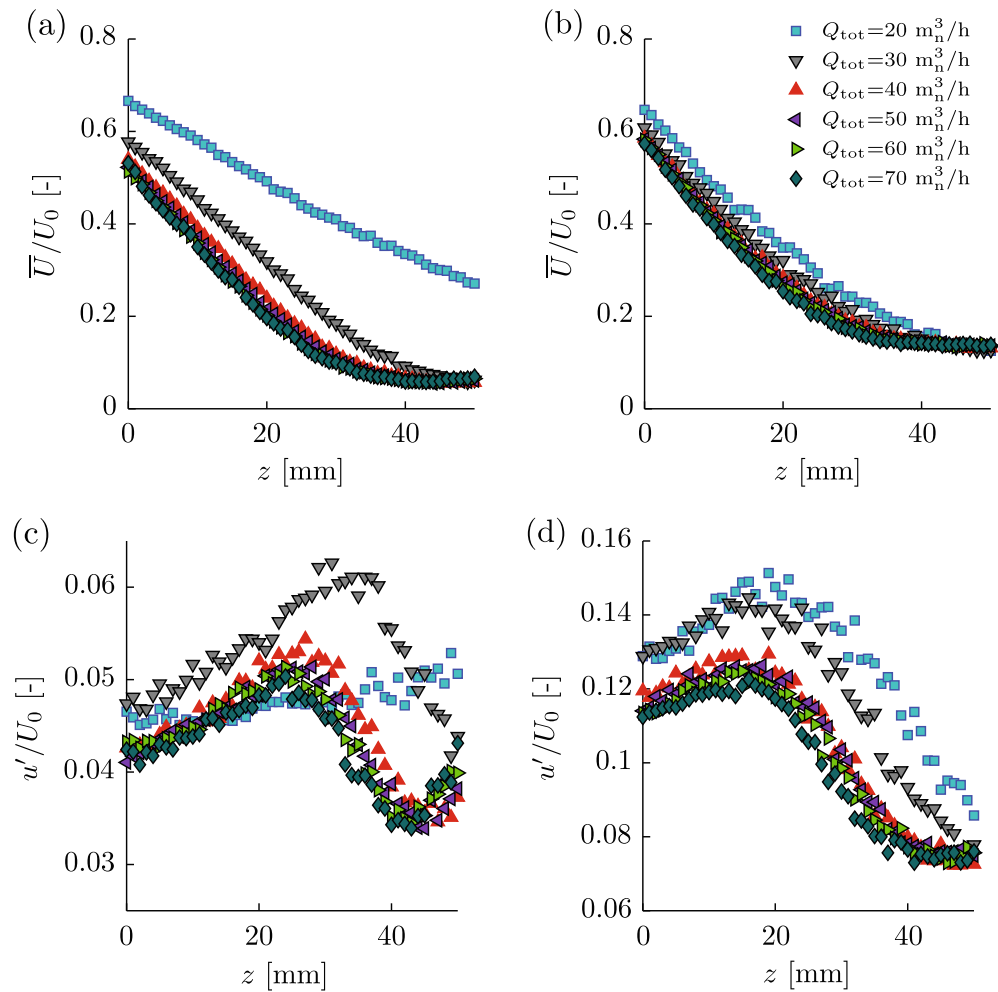

Fig. 7 Mean and rms velocity profiles on centerline at different bulk velocities; $U_{0}=3.9,5.9,7.8,9.8,11.8$, $13.7 \mathrm{~m} / \mathrm{s}$. a mean velocity of hexagonal grid, b mean velocity of fractal grid $\left(R_{\mathrm{t}}=0.29\right)$, c rms velocity of hexagonal grid and $\mathbf{d}$ rms velocity of fractal grid $\left(R_{\mathrm{t}}=0.29\right)$. The location $z=0$ is defined at the exit of the burner

between calculating the rms velocity at the centerline or averaging over a radius $(r=5 \mathrm{~mm})$ is insignificant to the conclusions as well.

The appropriate volumetric flow rate has been chosen according to Day et al. [32], who conclude that the flow field downstream of a low-swirl burner becomes self-similar if the averaged bulk velocity, $U_{0}$, is larger than a certain critical value. In order to upscale the results to industrial sizes, this flow self-similarity in the nearfield divergence region is crucial. Because Day et al. only use a hexagonal grid, self-similarity of the flow field has to be analyzed using fractal grids as well.

In Fig. 7, the normalized mean and rms velocity profiles are shown for both the hexagonal and the fractal $R_{\mathrm{t}}=0.29$ grid. As can be seen in Fig. 7a, the normalized mean velocity becomes self-similar for a bulk flow larger than $40 \mathrm{~m}_{\mathrm{n}}^{3} / \mathrm{h}$ (i.e. $U_{0} \geq 7.8 \mathrm{~m} / \mathrm{s}$ ). The same is valid for both fractal grids, as can be seen in Fig. 7b. The other fractal grid $\left(R_{\mathrm{t}}=0.40\right)$ shows a similar profile and is left out for economy of space. The normalized rms velocity profile for the hexagonal grid is shown in Fig. 7c. The normalized rms velocity profile shows a self similar profile from $40 \mathrm{~m}_{\mathrm{n}}^{3} / \mathrm{h}$ (i.e. $U_{0} \geq 7.8 \mathrm{~m} / \mathrm{s}$ ). The same is true for the normalized rms velocity profile using a fractal grid, as can be seen in Fig. $7 \mathrm{~d}$. This flow rate of $40 \mathrm{~m}_{\mathrm{n}}^{3} / \mathrm{h}$ will therefore be used as a minimum. In order to quantify a difference in flow fields between 
the grids, the normalized axial divergence rate, $a_{z}=\frac{1}{U_{0}} \cdot \frac{d \bar{U}}{d z}$, has been calculated according to [21]. Figure $7 \mathrm{a} \& \mathrm{~b}$ show that the nearfield divergence region, where the normalized axial divergence rate is constant, is at $x<25 \mathrm{~mm}$. The divergence rates are $0.0153,0.0148$ and 0.0161 for the hexagonal, $R_{\mathrm{t}}=0.29$ and $R_{\mathrm{t}}=0.40$ fractal grids respectively. This indicates that the overall flow field changes upon changing the grid. However, for the use of fractal grids in industry, similar overall flow fields are not a prerequisite. If necessary, these differences in divergence rates can be compensated for by using different swirl numbers.

\subsection{Measuring lean stability limit}

The lean stability limit is studied in order to determine how fractal grids influence the turndown ratio of a gas turbine. The lean stability limit refers to a state where the flame becomes unstable from the burner and the flame is physically blown off. The stability limit has been measured using blow-off experiments similar to [17]. Both the volumetric flow rate of reactants and the hydrogen ratio were maintained constant while the equivalence ratio was incrementally decreased, which results in a reduced flame speed. This causes the flame to stabilize more downstream (where the speed of the flow is lower) which ultimately leads to blow-off. After each decrement in equivalence ratio, a pause interval of approximately 10 $\mathrm{s}$ was used to check for instability. Each decrement in equivalence ratio was in the order of [0.004-0.01]. When blow-off occurred in the approximately 10 seconds, the flow conditions were considered as the lean stability limit. Due to the practicality for industry the lean stability limit results will be expressed in terms of the adiabatic flame temperature. Due to the stochastic nature of the blow-off event, a series of blow-off experiments were performed for a single grid/flow rate to obtain converged statistics. To calculate an appropriate sample size, 30 consecutive experiments were carried out, which revealed a normal distribution of the equivalence ratio at which blow-off occured. In order to estimate the mean value with a $95 \%$ confidence interval smaller than 0.01 (in equivalence ratio) a sample size of 10 is needed, according to "95\% confidence interval" theorem [33].

\section{Bouten Effect for Hydrogen Enrichment}

In this section, the results are shown of the beneficial combination of fractal grids and low-swirl combustion at $\phi=0.7$, using multiple blends of hydrogen-enriched natural gas. For natural gas, the additional turbulence of a fractal grid increases the local consumption speed while maintaining the characteristic linear relation, as shown by Verbeek et al. [8]. The main question here is whether hydrogen enrichment influences this effect. The molar based hydrogen fraction, defined as $n_{H_{2}}=X_{H_{2}} /\left(X_{H_{2}}+X_{\mathrm{NG}}\right)$, does not affect the flame shape in terms of $\bar{c}$ or the distribution of the flame surface, expressed in $\Sigma_{3 \mathrm{D}}$. Examples of $\bar{c}$ and $\Sigma_{3 \mathrm{D}}$ can be seen in [8]. Since $\bar{c}$ and $\Sigma_{3 \mathrm{D}}$ are hardly affected and are only intermediate results, we will only discuss the local consumption speed in more detail here.

In Fig. 8 the local consumption speed is plotted versus the rms velocity, both normalized with the laminar unstretched flame speed. Here, the color of the markers denote the three girds, where the gray markers denote the data points using the hexagonal grid, the black markers are used for the fractal grid with $R_{\mathrm{t}}=0.4$ and the white markers stand for the fractal grid with $R_{\mathrm{t}}=0.29$. The shape of the markers denote the bulk flow and hydrogen fraction. As recorded by Verbeek et al. [8], the flames using hexagonal grids stabilize more downstream and at lower rms velocities than flames using fractal grids. This stabilization height can be found in Table 1. A lower stabilization height allows for a smaller 


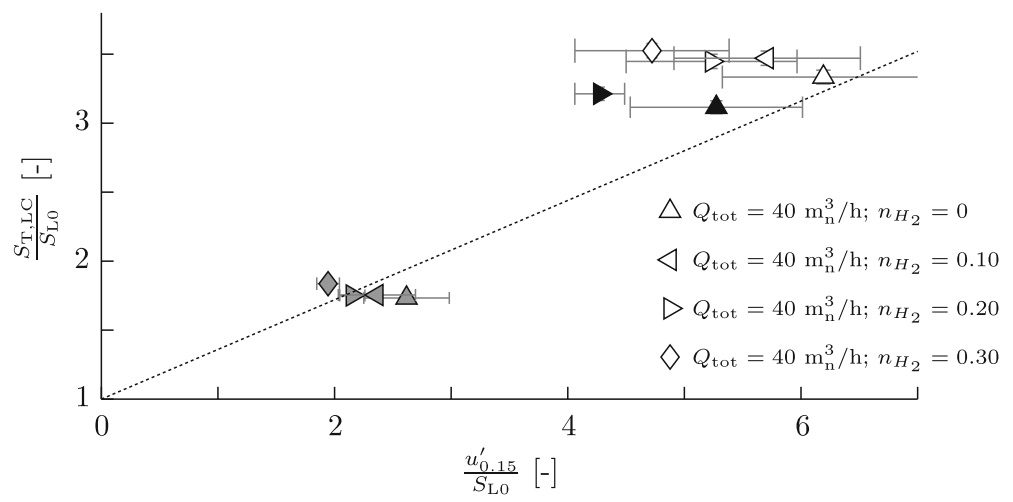

Fig. 8 Normalized local consumption speed, $S_{\mathrm{T}, \mathrm{LC}}$, versus the normalized rms velocity. The color of the markers denotes the three grids, where the hexagonal grid is gray, the fractal $\left(R_{\mathrm{t}}=0.4\right)$ grid is black and the fractal $\left(R_{\mathrm{t}}=0.29\right)$ grid is white. The shape of the markers denotes the flow rate and hydrogen fraction and these are given in the legend. The error bars in $S_{\mathrm{T}, \mathrm{LC}} / S_{\mathrm{L} 0}$ show the $95 \%$ confidence interval and in $u_{0.15}^{\prime} / S_{\mathrm{L} 0}$ are according to the estimated error of the hot-wire. The rms velocity is determined at $\bar{c}=0.15$

length of an industrial combustion chamber. The hydrogen fraction has no significant influence on the stabilization height. The change in $u_{0.15}^{\prime} / S_{\mathrm{L} 0}$ for varying $n_{H_{2}}$ is mainly due to the change in $S_{\mathrm{L} 0}$, as can be seen in Table $1 ; u_{0.15}^{\prime}$ is insensitive to the hydrogen fraction but $S_{\mathrm{L} 0}$ depends on $n_{H_{2}}$. The characteristic linear relation holds for natural gas, but also in the case of hydrogen enrichment. Cheng et al. [21] have already concluded that this characteristic linear relation holds for hydrogen-rich fuels, but with an increased linear constant. The same is shown in Fig. 8, where the linear relation fans out counter clockwise with increasing the hydrogen fraction. This means than increasing the hydrogen fraction increases the normalized local consumption speed for each grid. The linear trend (dashed line), which links the pure natural gas cases, has a linear coefficient that is lower than recorded by

Table 1 Stabilization height, rms velocity at the flame brush and laminar unstretched flame speed at $\phi=0.7$

\begin{tabular}{llllll}
\hline & $\begin{array}{l}R_{\mathrm{t}} \\
{[-]}\end{array}$ & $\begin{array}{l}n_{\mathrm{H}_{2}} \\
{[-]}\end{array}$ & $\begin{array}{l}z_{0.15} \\
{[\mathrm{~mm}]}\end{array}$ & $\begin{array}{l}u_{0.15}^{\prime} \\
{[\mathrm{m} / \mathrm{s}]}\end{array}$ & $\begin{array}{l}S_{\mathrm{L} 0} \\
{[\mathrm{~m} / \mathrm{s}]}\end{array}$ \\
\hline Hexagonal & - & 0.00 & 25 & 0.44 & 0.17 \\
Hexagonal & - & 0.10 & 24 & 0.44 & 0.18 \\
Hexagonal & - & 0.20 & 22 & 0.43 & 0.20 \\
Hexagonal & - & 0.30 & 22 & 0.43 & 0.22 \\
Fractal & 0.40 & 0.00 & 16 & 0.89 & 0.17 \\
Fractal & 0.40 & 0.20 & 14 & 0.85 & 0.20 \\
Fractal & 0.29 & 0.00 & 15 & 1.05 & 0.17 \\
Fractal & 0.29 & 0.10 & 14 & 1.05 & 0.18 \\
Fractal & 0.29 & 0.20 & 13 & 1.05 & 0.20 \\
Fractal & 0.29 & 0.30 & 12 & 1.05 & 0.22 \\
\hline
\end{tabular}

The variable $z_{0.15}$ is the flow direction coordinate at which $\bar{c}=0.15 . u_{0.15}^{\prime}$ is the rms velocity at which $\bar{c}=0.15 . S_{\mathrm{L} 0}$ has been calculated using CHEMKIN PREMIX and the GRI 3.0 reaction mechanism 
Verbeek et al. [8], 0.36 versus 0.47 respectively. This is most probably due to the overprediction of the parameterizing method versus the integration method, as explained in Section 2.1.3. To estimate the effect that the true, non-unity stretch factor would have on the results, an empirical equation of Bray and Cant [34] has been used. Bray and Cant introduce a simplified formula derived from direct numerical simulations that state that $I_{0}$ is proportional with the Lewis and Markstein numbers. The Lewis number relates the thermal and mass diffusivity and the Markstein number characterizes the effect of local heat release on variations in the surface topology, i.e., stretch and strain effects [35]. Therefore, a larger $n_{H_{2}}$ results, via an increase in Lewis number, in a larger $S_{\mathrm{T}, \mathrm{LC}} / S_{\mathrm{L} 0}$. Because the Markstein number depends on the Lewis number [36], a larger $u_{0.15}^{\prime}$ results in higher strains, which means an increase of $I_{0}$ upon hydrogen enrichment. This also results in an increase in $S_{\mathrm{T}, \mathrm{LC}} / S_{\mathrm{L} 0}$, via Eq. 1 . Therefore, cases with a large $n_{H_{2}}$ and $u_{0.15}^{\prime}$ have a relatively high $I_{0}$. This implies that the stretch factor alters individual results, but the overall trend and therefore conclusions remain unaltered.

\section{Effect on Lean Stability Limit}

In this section, the lean stability limit for multiple hydrogen fractions and flow rates is investigated. Therefore, the adiabatic flame temperature in this section is not constant, but for each case a different adiabatic flame temperature at this lean stability limit is found and studied. These lean stability limits are determined in Section 4.1. Secondly, the shape of the flame is discussed in Section 4.2. Thirdly, the main cause of the changes in the lean stability limit is discussed in Section 4.3 and the local consumption speed is determined in Section 4.4.

\subsection{Determination of lean stability limit}

The lean stability limit, $T_{\text {ad,bo }}$, is required to analyze the influence of fractal-grid-generated turbulence on the turn-down ratio and it has been determined by periodically decreasing the adiabatic flame temperature (or equivalence ratio), as described in Section 2.3. The result is shown in Fig. 9, which shows the lean stability limit for (a) a varying hydrogen fraction and (b) a varying flow rate. The lean stability limit of a low-swirl flame with a hexagonal grid (O) for pure natural gas is approximately $1676 \mathrm{~K}$. Changing to a fractal grid increases this value slightly to $1705 \mathrm{~K}$ and $1728 \mathrm{~K}$ for $R_{\mathrm{t}}=0.40(\boldsymbol{\nabla})$ and $R_{\mathrm{t}}=0.29(\triangle)$ respectively, see Fig. 9a. Increasing the hydrogen fraction to $n_{H_{2}}=0.30$ decreases the lean stability limit for all cases linearly. For the hexagonal grid, this results in a lean stability limit of approximately $1448 \mathrm{~K}$. The mutual difference between the hexagonal and fractal did not change significantly upon hydrogen enrichment. A higher lean stability limit represents a decrease of the turn-down ratio of a combustor, which is unwanted. This means that the hexagonal grid has a slightly higher turn-down ratio, for all investigated hydrogen fractions. The two data points at $Q_{\text {tot }}=40 \mathrm{~m}_{\mathrm{n}}^{3} / \mathrm{h}$ in Fig. $9 \mathrm{~b}$ correspond with the two data points at $n_{H_{2}}=0.10$ in Fig. 9a. As can be seen in Fig. 9b, increasing the flow rate significantly affects the lean stability limit; the hexagonal grid shows an exponential-like increase in $T_{\text {ad,bo }}$ with increasing bulk flow. The fractal grid also shows this exponential-like increase, and the increase is larger than the increase when using the hexagonal grid. Although fractal grids cause a more compact combustion in the center of the flame, even at hydrogen fractions up to $n_{H_{2}}=0.30$, the stability of the flame appears to be worse than by using hexagonal grids. 

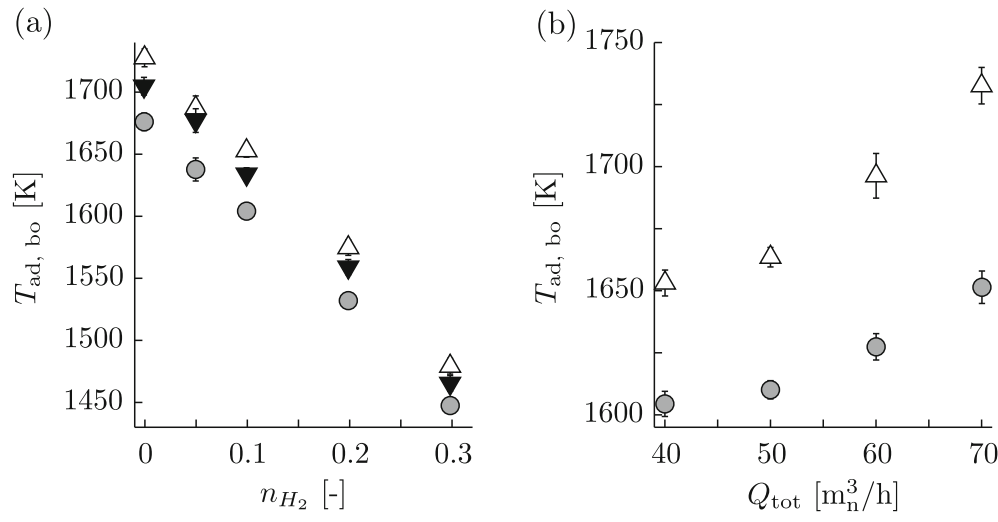

Fig. 9 Lean stability limit based on 10 experiments for a variable hydrogen enrichment at constant flow rate $\left(Q_{\text {tot }}=40 \mathrm{~m}_{\mathrm{n}}^{3} / \mathrm{h}\right)$ and $\mathbf{b}$ variable flow rate at constant hydrogen fraction $\left(n_{H_{2}}=0.10\right)$. The data is shown for three grids: $O$ Hexagonal grid, $\boldsymbol{\nabla}$ fractal $\left(R_{\mathrm{t}}=0.4\right)$ grid and $\Delta$ fractal $\left(R_{\mathrm{t}}=0.29\right)$ grid. The error bars show the $95 \%$ confidence interval for each case

\subsection{Flame shape at lean stability conditions}

The mean progress variable, as shown in Fig. 10, is calculated to analyze the flame shape and settling position. Figure $10 \mathrm{a} \& \mathrm{~b}$ show the pure natural gas cases for the hexagonal and fractal grid respectively and Fig. 10c \& d show the same grids for $n_{H_{2}}=0.30$. The comparison of these cases shows that the shape of the flame in terms of $\bar{c}$ is similar for the same grid. This applies to both the hexagonal and fractal grid. Hydrogen enrichment of up to $30 \%$ does not significantly change the shape of the flame. This can also be seen in the stabilization height (see Table 2), which is quite constant for a single grid. In Fig. 10b, the maximum mean progress variable that is reached for the fractal grid is approximately 0.8 .
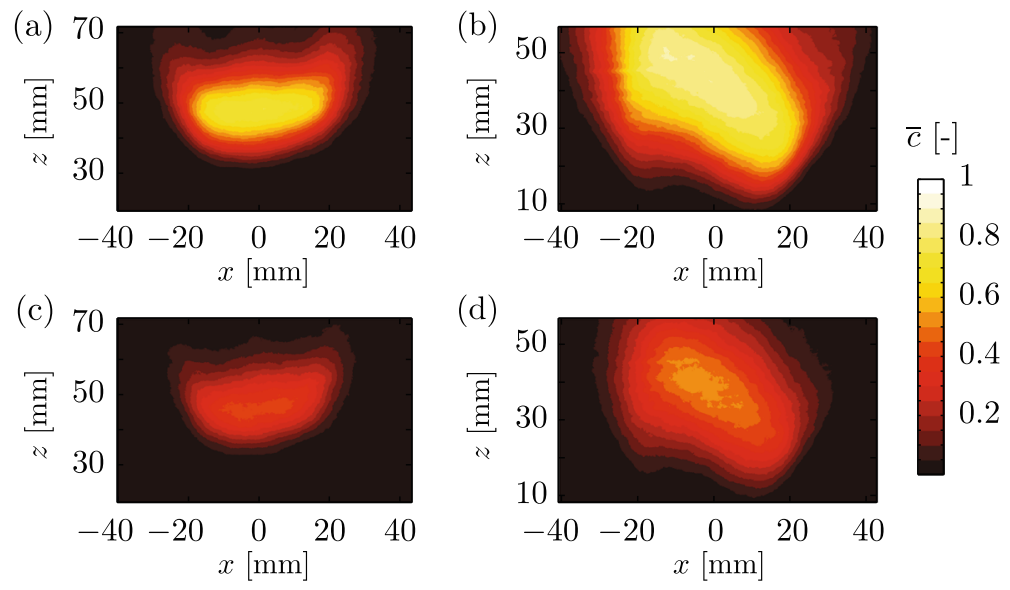

Fig. 10 Mean progress variable for: a Hexagonal $n_{H_{2}}=0$, b Fractal $R_{\mathrm{t}}=0.29$ and $n_{H_{2}}=0$, c Hexagonal $n_{H_{2}}=0.30$ and $\mathbf{d}$ Fractal $R_{\mathrm{t}}=0.29$ and $n_{H_{2}}=0.30$ at constant flow rate $\left(Q_{\text {tot }}=40 \mathrm{~m}_{\mathrm{n}}^{3} / \mathrm{h}\right) \cdot z=0 \mathrm{~mm}$ is at the exit of the burner. The data is shown at the lean stability limit 
Table 2 Stabilization height, rms velocity at the flame brush and laminar unstretched flame speed at $T_{\text {ad,bo }}$

\begin{tabular}{llllll}
\hline & $\begin{array}{l}n_{H_{2}} \\
{[-]}\end{array}$ & $\begin{array}{l}Q_{\text {tot }} \\
{\left[\mathrm{m}_{\mathrm{n}}^{3} / \mathrm{h}\right]}\end{array}$ & $\begin{array}{l}z_{0.15} \\
{[\mathrm{~mm}]}\end{array}$ & $\begin{array}{l}u_{0.15}^{\prime} \\
{[\mathrm{m} / \mathrm{s}]}\end{array}$ & $\begin{array}{c}S_{\mathrm{L} 0} \\
{[\mathrm{~m} / \mathrm{s}]}\end{array}$ \\
\hline Hexagonal & 0.00 & 40 & 34 & 0.40 & 0.11 \\
Hexagonal & 0.05 & 40 & 34 & 0.40 & 0.10 \\
Hexagonal & 0.10 & 40 & 34 & 0.40 & 0.09 \\
Hexagonal & 0.20 & 40 & 34 & 0.40 & 0.06 \\
Hexagonal & 0.30 & 40 & 34 & 0.40 & 0.04 \\
Hexagonal & 0.10 & 50 & 34 & 0.46 & 0.09 \\
Hexagonal & 0.10 & 60 & 33 & 0.51 & 0.09 \\
Hexagonal & 0.10 & 70 & 31 & 0.62 & 0.10 \\
Fractal & 0.00 & 40 & 14 & 1.05 & 0.13 \\
Fractal & 0.05 & 40 & 14 & 1.05 & 0.12 \\
Fractal & 0.10 & 40 & 14 & 1.05 & 0.11 \\
Fractal & 0.20 & 40 & 14 & 1.05 & 0.08 \\
Fractal & 0.30 & 40 & 14 & 1.05 & 0.05 \\
Fractal & 0.10 & 50 & 14 & 1.23 & 0.11 \\
Fractal & 0.10 & 60 & 14 & 1.46 & 0.12 \\
Fractal & 0.10 & 70 & 13 & 1.64 & 0.14 \\
\hline
\end{tabular}

$z_{0.15}$ is the flow direction coordinate at which $\bar{c}=0.15 \bar{c}_{\max } \cdot u_{0.15}^{\prime}$ is the rms velocity at the location where $\bar{c}=0.15 \bar{c}_{\max } . S_{\mathrm{L} 0}$ has been calculated using CHEMKIN PREMIX and the GRI 3.0 reaction mechanism

This means that approximately $80 \%$ of the time that region is classified as burned. Increasing the hydrogen fraction clearly reduces this maximum. The same is true for the hexagonal grid. The fractal case shows an asymmetric $\bar{c}$ profile. Increasing the hydrogen fraction does not affect this phenomena. Lastly, Fig. 10 also shows that the flame of fractal grids stabilize more upstream. Only four cases are shown here for economy of space, because other cases show intermediate results.

Between the grids there is considerable more variation, which is due to differences in flow-field. A somewhat $\mathrm{W}$-shaped flame can be recognized for the fractal grid case near blow-off, see Fig. 10b or d. Such a shape indicates that the flame is also stabilized by the shear layer between the inner core and the outer swirling flow [37]. A next step would be to use PIV to determine more precisely the difference in flow field and optimize the design of the fractal grid to properly stabilize a low-swirl flame. However, this is left for further research.

\subsection{Cause of the change in lean stability limit}

Previously, the effect that parameter changes (either in $n_{H_{2}}$ or $Q_{\text {tot }}$ ) had on the lean stability limit was shown. In this section, the aim is to combine this data and attribute the variations to a single variable. In Fig. 11, the lean stability limit versus the rms velocity at the flame brush is shown, where the latter is determined at $\bar{c}=0.15 \bar{c}_{\text {max }}$. As discussed in Section 2.2, the influence of this definition does not change the results. In this figure, three parameters $\left(Q_{\text {tot }}\right.$, $n_{H_{2}}$ and the grid) are varied and these are visually represented with gray arrows. Increasing the hydrogen ratio does not seem to affect the rms velocity, because the flame settles at more 


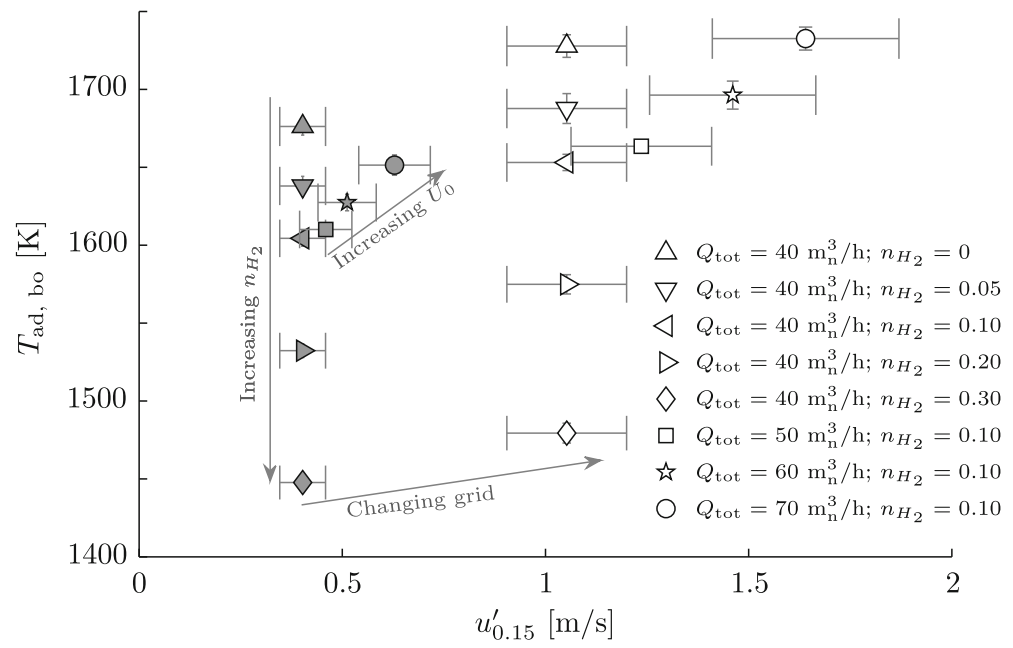

Fig. 11 Lean stability limit versus rms velocity. The color of the markers denotes the two grids, where the hexagonal grid is gray and the fractal $R_{\mathrm{t}}=0.29$ grid is white. The shape of the markers denotes the flow rate and hydrogen fraction and these are given in the legend. The error bars in $T_{\text {ad,bo }}$ show the $95 \%$ confidence interval based on 10 experiments and the error bars in $u_{0.15}^{\prime} / S_{\mathrm{L} 0}$ show the estimated error of the hot-wire. The rms velocity is determined at the location where $\bar{c}=0.15 \bar{c}_{\max }$. Three main parameter changes are shown with gray arrows: increasing the hydrogen fraction, increasing the bulk flow and changing the grid from hexagonal to fractal

or less the same height, see Table 2. However, changing the grid, while keeping the bulk flow and hydrogen fraction constant, increases the rms velocity at the flame as well as the lean stability limit. This applies to all investigated conditions. The same holds for changing $Q_{\text {tot}}$; an increased flow rate, while keeping the other parameters constant, increases both $T_{\text {ad,bo }}$ and $u_{0.15}^{\prime}$. Changes in parameters that result in a higher rms velocity at the flame brush, result in a higher lean stability limit.

\subsection{Local consumption speed at lean flame stability conditions}

The local consumption speed has been calculated as described in Section 2.1.3. Visually, $n_{\mathrm{H}_{2}}$ does not affect the distribution of the flame surface, only the total quantity differs. In Fig. 12 the local consumption speed is plotted versus the rms velocity at the flame brush, both are normalized with the laminar unstretched flame speed. Here, the gray markers stand for the data points using the hexagonal grid and the white markers are used for the fractal grid with $R_{\mathrm{t}}=0.29$. A clear distinction is made in local consumption speed between the grids; the hexagonal grid has a significantly lower $S_{\mathrm{T}, \mathrm{LC}} / S_{\mathrm{L} 0}$ compared to the fractal grid. The observed $u_{0.15}^{\prime} / S_{\mathrm{L} 0}$ values here are a lot higher than recorded in Section 3 due to the low $S_{\mathrm{L} 0}$ values, as denoted in Table 2. A linear trend can be seen for most points. Such a linear trend is characteristic for a low-swirl burner [7, 8, 38], as was shown in this study for $\phi=0.7$ in Section 3. However, here all cases are at different equivalence ratio, which has an effect on the laminar flame speed, as shown in Table 2. The expected fanning effect due to a higher linear coefficient upon hydrogen enrichment is not observed. Moreover, the highest hydrogen additions $\left(n_{H_{2}}=0.30\right)$ show a large deviating from this expectation and this is most probably due to the under-prediction of the stretch factor, as discussed in Section 3. It is unlikely that the increase of $u_{0.15}^{\prime} / S_{\mathrm{L} 0}$ for high hydrogen fractions is related 


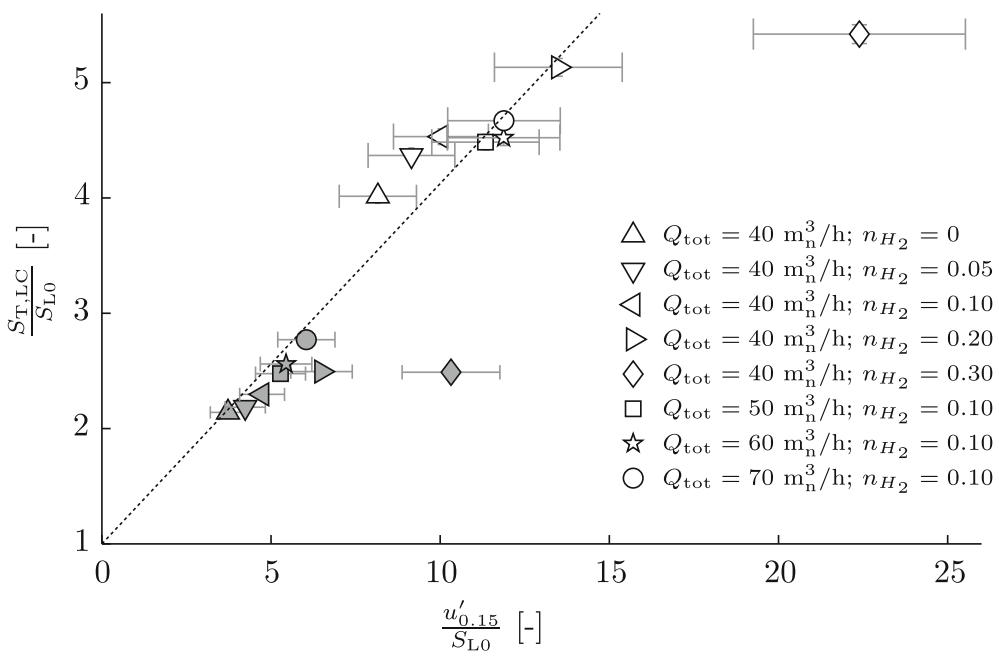

Fig. 12 Normalized local consumption speed, $S_{\mathrm{T}, \mathrm{LC}}$, versus the normalized rms velocity. The color of the markers denotes the two grids, where the hexagonal grid is gray and the fractal $R_{\mathrm{t}}=0.29$ grid is white. The shape of the markers denotes the flow rate and hydrogen fraction and these are given in the table. The error bars in $S_{\mathrm{T}, \mathrm{LC}} / S_{\mathrm{L} 0}$ show the $95 \%$ confidence interval and in $u_{0.15}^{\prime} / S_{\mathrm{L} 0}$ is according to the estimated error of the hot-wire. Rms velocity is determined at the location where $\bar{c}=0.15 \bar{c}_{\max }$

to the appearance of diffusive thermal instabilities, because no increase of $u_{0.15}^{\prime} / S_{\mathrm{L} 0}$ was registered at $\phi=0.7$ (actually, here $u_{0.15}^{\prime} / S_{\mathrm{L} 0}$ decreases upon hydrogen enrichment). As said before, the under-prediction of $I_{0}$ only changes individual results and not the overall trend and conclusions.

The linear coefficient in Fig. 12 is 0.31 , which is quite smaller than the linear coefficient for the data at $\phi=0.7$, which is 0.36 . This is most probably due to the under-predictions of $S_{\mathrm{T}, \mathrm{LC}} / S_{\mathrm{L} 0}$ by integrating over part of the flame, as explained in Section 2.1.3.

\section{Conclusions and Outlook}

The use of fractal grids to enhance the limited reaction rate in the center of a low-swirl flame has been demonstrated in the past. To test if this technique is also useful at more industrial conditions, additional research has been performed. This research focused on two different aspects; the benefit of fractal grids when combusting alternative fuels and the effect of fractal grids on the lean stability limit. These alternative fuels have been simulated by hydrogen-enriched natural gas. The turbulence-generating grids that were investigated are constructed using an iterative pattern of crosses. The thickness ratio of the consecutive crosses, $R_{\mathrm{t}}$, is constant and two fractal grids have been studied $\left(R_{\mathrm{t}}=0.29\right.$ and 0.40$)$. These have been compared with a commonly used grid which consists of perforated plate with openings in a hexagonal pattern, i.e., a hexagonal grid. OH-LIF experiments were carried out to quantify the local consumption speed, $S_{\mathrm{T}, \mathrm{LC}}$, by evaluating the flame surface density and mean progress variable. The increase in $S_{\mathrm{T}, \mathrm{LC}}$ is linear in the rms velocity at the flame brush, $u_{0.15}^{\prime}$, which is in correspondence with literature [38]. The linear coefficient in this characteristic relation increases with hydrogen enrichment, which corresponds with observations of others as well [21]. The investigated hydrogen fractions $\left(0 \leq n_{H_{2}} \leq 0.3\right)$ do not 
otherwise influence the relation between the increase in $u_{0.15}^{\prime}$ by fractal grids and the local consumption speed. Fractal grids enhance the turbulence in the low-swirl burner, irrespective of the hydrogen fraction. It was found that $u_{0.15}^{\prime}$ is about two times higher for the fractal grid, compared to the hexagonal grid. Although the turbulence at the flame front has not been measured directly by hot-wire anemometry, a reliable estimate has been made using the data from [7]. The blow-off experiments allow for an evaluation of the lean stability limit, $T_{\text {ad,bo }}$. Compared to the hexagonal grid, fractal grids show an increase in $T_{\text {ad,bo }}$, where $R_{\mathrm{t}}=0.29$ has the highest increase (of $50 \mathrm{~K}$ compared to the hexagonal grid). Increasing $n_{\mathrm{H}_{2}}$ from 0 to 0.3 results in more than $200 \mathrm{~K}$ decrease of $T_{\text {ad,bo }}$ for all grids. Increasing the flow rate, from 40 to $70 \mathrm{~m}_{\mathrm{n}}^{3} / \mathrm{h}$, increases $T_{\text {ad,bo }}$ exponential-like for both the hexagonal and the fractal $\left(R_{\mathrm{t}}=0.29\right)$ grid, where the fractal grid has a more rapid increase. In all of the considered cases, a positive correlation was found between $T_{\text {ad,bo }}$ and $u_{0.15}^{\prime}$; all changes that increased the rms velocity at the flame brush, resulted in an increase in the lean stability limit. Additionally, $S_{\mathrm{T}, \mathrm{LC}}$ has been determined at $T_{\mathrm{ad}, \mathrm{bo}}$. The characteristic linear relation between $S_{\mathrm{T}, \mathrm{LC}} / S_{\mathrm{L} 0}$ and $u_{0.15}^{\prime} / S_{\mathrm{L} 0}$ remains, while the adiabatic flame temperature is changed significantly and the flame is at its lean stability limit. This indicates that low-swirl combustion gives a predictable turbulent flame speed, usable for the whole operability range. This study has indicated that fractal grids and a low-swirl burner are a beneficial combination. Although, only experiments with up to $30 \%$ hydrogen addition were performed, there is no indication (yet) that this breaks down at higher $n_{H_{2}}$. The reduced stability of the flame when increasing the turbulence (either by a higher flow rate or by applying fractal grids) asks for more research. Here, a single swirler design is used, while at higher turbulence levels a different swirler (and swirl number) might be favorable. This was a preliminary study, using a lab-scale combustor in ambient conditions and a next step would be to test at an industrial-scale, including preheating and pressurizing.

Open Access This article is distributed under the terms of the Creative Commons Attribution 4.0 International License (http://creativecommons.org/licenses/by/4.0/), which permits unrestricted use, distribution, and reproduction in any medium, provided you give appropriate credit to the original author(s) and the source, provide a link to the Creative Commons license, and indicate if changes were made.

\section{References}

1. Yegian, D., Cheng, R.: Development of a lean premixed low-swirl burner for low NOx practical applications. Combust. Sci. Technol. 139(1), 207-227 (1998)

2. Cheng, R.K., Yegian, D.T., Miyasato, M.M., Samuelsen, G.S., Benson, C.E., Pellizzari, R., Loftus, P.: Scaling and development of low-swirl burners for low-emission furnaces and boilers. Proc. Combust. Inst. 28(1), 1305-1313 (2000)

3. Johnson, M.R., Littlejohn, D., Nazeer, W.A., Smith, K.O., Cheng, R.K.: A comparison of the flowfields and emissions of high-swirl injectors and low-swirl injectors for lean premixed gas turbines. Proc. Combust. Inst. 30(2), 2867-2874 (2005)

4. Chan, C.K., Lau, K.S., Chin, W.K., Cheng, R.K.: Freely propagating open premixed turbulent flames stabilized by swirl. Proc. Combust. Inst. 24(1), 511-518 (1992)

5. Bedat, B., Cheng, R.K.: Experimental study of premixed flames in intense isotropic turbulence. Combust. Flame 100(3), 485-494 (1995)

6. Plessing, T., Kortschik, C., Peters, N., Mansour, M.S., Cheng, R.K.: Measurements of the turbulent burning velocity and the structure of premixed flames on a low-swirl burner. Proc. Combust. Inst. 28(1), 359-366 (2000)

7. Cheng, R.K., Nazeer, W., Smith, K., Littlejohn, D.: Laboratory studies of the flow field characteristics of low-swirl injectors for adaptation to fuel-flexible turbines. J. Eng. Gas Turb. Power 130(2), 10 (2008) 
8. Verbeek, A.A., Bouten, T.W.F.M., Stoffels, G.G.M., Geurts, B.J., van der Meer, T.H.: Fractal turbulence enhancing low-swirl combustion. Combust. Flame 162(1), 129-143 (2015)

9. Geipel, P., Goh, K.H.H., Lindstedt, R.P.: Fractal-generated turbulence in opposed jet flows. Flow Turbul. Combust. 85(3-4), 397-419 (2010)

10. Soulopoulos, N., Kerl, J., Sponfeldner, T., Beyrau, F., Hardalupas, Y., Taylor, A.M.K.P., Vassilicos, J.C.: Turbulent premixed flames on fractal-grid-generated turbulence. Fluid Dyn. Res. 45(6), 061404 (2013)

11. Hurst, D., Vassilicos, J.C.: Scalings and decay of fractal-generated turbulence. Phys. Fluids 19, 035103 (2007)

12. Coffey, C.J., Hunt, G.R., Seoud, R.E., Vassilicos, J.C.: Mixing effectiveness of fractal grids for inline static mixers. Proof of concept report for the attention of Imperial Innovations, Imperial College London (2007). https://workspace.imperial.ac.uk/tmfc/public/proof_FG.PDF

13. Abou El-Azm Aly, A., Chong, A., Nicolleau, F.C.G.A., Beck, S.B.M.: Experimental study of the pressure drop after fractal-shaped orifices in turbulent pipe flows. Exp. Therm. Fluid Sci. 34(1), 104-111 (2010)

14. Keylock, C.J., Nishimura, K., Nemoto, M., Ito, Y.: The flow structure in the wake of a fractal fence and the absence of an inertial regime. Environ. Fluid Mech. 12(3), 227-250 (2012)

15. Mazellier, N., Vassilicos, J.C.: Turbulence without Richardson-Kolmogorov cascade. Phys. Fluids 22(7), $1-25$ (2010)

16. Todd, D.M.: Gas turbine improvements enhance IGCC viability. Gasification Technologies Conference October 8-11, San Fransisco (2000)

17. Schefer, R.W., Wicksall, D.M., Agrawal, A.K.: Combustion of hydrogen-enriched methane in a lean premixed swirl-stabilized burner. Proc. Combust. Inst. 29(1), 843-851 (2002)

18. Cheng, R.K., Levinsky, H.: Chapter 6 - lean premixed burners. In: Dunn-Rankin, D. (ed.) Lean combustion, pp. 161-177. Academic, Burlington (2008)

19. Schefer, R.W.: Hydrogen enrichment for improved lean flame stability. Int. J. Hydrogen Energy 28(10), 1131-1141 (2003)

20. Littlejohn, D., Cheng, R.K.: Fuel effects on a low-swirl injector for lean premixed gas turbines. Proc. Combust. Inst. 31(1), 3155-3162 (2007)

21. Cheng, R.K., Littlejohn, D., Strakey, P.A., Sidwell, T.: Laboratory investigations of a low-swirl injector with $\mathrm{H} 2$ and CH4 at gas turbine conditions. Proc. Combust. Inst. 32(2), 3001-3009 (2009)

22. Littlejohn, D., Cheng, R.K., Noble, D.R., Lieuwen, T.: Laboratory investigations of low-swirl injectors operating with syngases. J. Eng. Gas Turb. Power 132(1), 011502 (2009)

23. Verbeek, A.A., Jansen, W., Stoffels, G.G.M., van der Meer, T.H.: Improved flame front curvature measurements for noisy OH-LIF images. In: Proceedings 8th World Conferences on Experimental Heat Transfer, Fluid Mechanics and Thermodynamics, ExHFT-8 (2013). http://doc.utwente.nl/86288/

24. Smith, G.P., Golden, D.M., Frenklach, M., Moriarty, N.W., Eiteneer, B., Goldenberg, M., Bowman, C.T., Hanson, R.K., Song, S., Gardiner, W.C., Jr., Lissianski, V.V., Qin, Z.: Gri-mech 3.0. http://www.me. berkeley.edu/gri_mech/

25. Veynante, D., Lodato, G., Domingo, P., Vervisch, L., Hawkes, E.R.: Estimation of three-dimensional flame surface densities from planar images in turbulent premixed combustion. Exp. Fluids 49(1), 267278 (2010)

26. Driscoll, J.F.: Turbulent premixed combustion: flamelet structure and its effect on turbulent burning velocities. Prog. Energ. Combust. 34(1), 91-134 (2008)

27. Kariuki, J., Dawson, J.R., Mastorakos, E.: Measurements in turbulent premixed bluff body flames close to blow-off. Combust. Flame 159(8), 2589-2607 (2012)

28. Day, M., Tachibana, S., Bell, J., Lijewski, M., Beckner, V., Cheng, R.K.: A combined computational and experimental characterization of lean premixed turbulent low swirl laboratory flames ii. hydrogen flames. Combust. Flame 162(5), 2148-2165 (2015)

29. Verbeek, A.A., Pos, R.C., Stoffels, G.G.M., Geurts, B.J., van der Meer, T.H.: A compact active grid for stirring pipe flow. Exp Fluids 54(10) (2013)

30. Pope, S.B.: Turbulent flows. Cambridge University Press, Cambridge (2000)

31. Swaminathan, M.K., Rankin, G.W., Sridhar, K.: Evaluation of the basic systems of equations for turbulence measurements using the monte carlo technique. J. Fluid Mech. 170, 1-19 (1986)

32. Day, M., Tachibana, S., Bell, J., Lijewski, M., Beckner, V., Cheng, R.K.: A combined computational and experimental characterization of lean premixed turbulent low swirl laboratory flames: I. methane flames. Combust. Flame 159(1), 275-290 (2012)

33. Montgomery, D.C., Runger, G.C. Applied statistics and probability for engineers, 5th edn. Wiley, Asia Pte Ltd (2011)

34. Bray, K.N.C., Cant, R.S.: Some applications of Kolmogorov's turbulence research in the field of combustion. Proc. R. Soc. Lond. A 434(1890), 217-240 (1991) 
35. Poinsot, T., Veynante, D. Theoretical and numerical combustion, 3rd edn. Aquaprint, France (2001)

36. Ai, Y., Zhou, Z., Chen, Z., Kong, W.: Laminar flame speed and Markstein length of syngas at normal and elevated pressures and temperatures. Fuel 137, 339-345 (2014)

37. Ballachey, G.E., R., J.M.: Prediction of blowoff in a fully controllable low-swirl burner burning alternative fuels: effects of burner geometry, swirl, and fuel composition. Proc. Combust. Inst. 34(2), 3193 3201 (2013)

38. Shepherd, I., Cheng, R.K.: The burning rate of premixed flames in moderate and intense turbulence. Combust. Flame 127(3), 2066-2075 (2001) 